\title{
HFC Access Network Design for Switched Broadcast TV Services
}

\author{
Tim Wauters, Jeffrey De Bruyne, Luc Martens, Didier Colle, Bart Dhoedt, Piet Demeester, and Kurt Haelvoet
}

\begin{abstract}
The Hybrid Fiber Coax (HFC) is a modern broadband access network, providing advanced interactive services such as Internet access, digital interactive television and cable telephony. The edge costs dominate the cost of launching new services, at the time of deployment (CapEx) as well as for maintenance $(\mathrm{OpEx})$. Therefore next-generation edge devices aim at the convergence of these services and their platforms into a single multi-service infrastructure, thus sharing resources and bandwidth. The focus of this paper is on the HFC access network design for bandwidth-intensive TV services, bringing standard as well as switched broadcast technologies into play. Standard broadcast TV service deployments, offered through the Digital Video Broadcast (DVB) platform, require low installation costs, but the bandwidth consumption increases linearly with the number of available TV channels, even for very unpopular channels. Switched broadcast TV channels, offered on the (Euro-) DOCSIS platform together with other interactive unicast services such as Video on Demand, are only streamed to a node on the HFC access network if they are requested locally, thus limiting bandwidth consumption for less popular channels. We present an access network design tool, based on an analytical TV traffic model, minimizing the installation cost on the edge devices and study the influence of the most important traffic and content parameters.
\end{abstract}

Index Terms-Digital TV, HFC, network convergence, switched broadcast.

\section{INTRODUCTION}

$\mathbf{T}$ HE CURRENT trend in broadband cable access networks is to converge towards one technological platform for Internet and data services, such as the (Euro-)DOCSIS standard for cable networks, and one for digital broadcast television services, such as the European DVB platform. The main reasons for this phenomenon are on one hand the integration of different interactive broadband services at the end user devices and on the other hand the ability for the service provider to enable dynamic bandwidth sharing among these services. This way, the dominant deployment costs from service-specific edge devices, in terms of both capital (CapEx) and operational expenditures (OpEx), can be reduced. A "many services, one network" model in which a single network can support all existing and novel services, dramatically reduces the total cost of ownership and the time to market for service providers [1].

Bandwidth sharing at the edge QAM devices on a HFC access network can be achieved by dynamically allocating the downstream spectrum, depending on fluctuations in user demand, in-

Manuscript received August 8, 2006; revised February 9. 2006. This work was supported in part by the IBBT-CIcK project.

T. Wauters, J. De Bruyne, L. Martens, D. Colle, B. Dhoedt, and P. Demeester are with the Department of. Information Technology (INTEC), Ghent University, B-9050 Gent, Belgium (e-mail: tim.wauters@intec.ugent.be).

K. Haelvoet is with Telenet, Access Engineering \& Service Integration, B-2800 Mechelen, Belgium.

Color versions of one or more of the figures in this paper are available online at http://ieeexplore.ieee.org.

Digital Object Identifier 10.1109/TBC.2007.894950

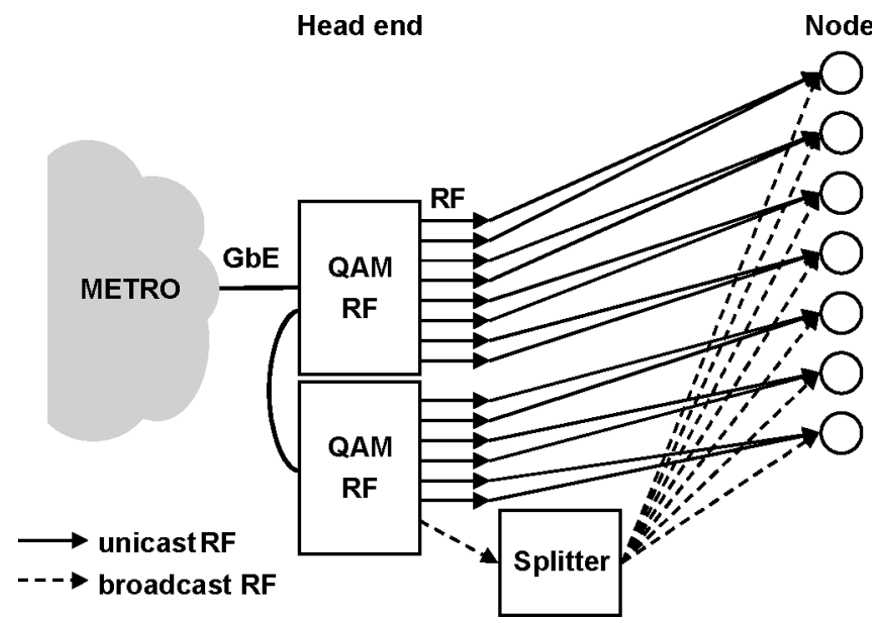

Fig. 1. Typical HFC access network configuration: Unicast and broadcast RF traffic from the head end to the optical nodes.

stead of using an inefficient fixed channel allocation, based on peak traffic for each single service. Another option is to reduce peak traffic bandwidth requirements on a per-service basis by making efficient use of the available unicast and broadcast technologies. For example, video on demand ( $\mathrm{VoD})$ services for very popular videos can be served through near $\mathrm{VoD}(\mathrm{nVoD})$ broadcast, instead of the unicast interactive $\mathrm{VoD}$ (iVoD), to combine multiple requests [2].

This paper presents a method to decrease the bandwidth spectrum usage for broadcast TV, by serving less popular channels through (unicast) switched broadcast on the access network, instead of standard broadcast technologies [3].

To explain the use of both technologies, a typical Hybrid Fiber Coax (HFC) access network configuration for unicast as well as broadcast services is shown in Fig. 1.

Transport networks for the delivery of triple-play services are evolving towards Internet Protocol over Gigabit Ethernet over Wavelength Division Multiplexing (IP/GbE/WDM) networks. At the edge of the metro network, GbE signals reach the head ends (HE), where the MPEG-2 encoded TV channels are multiplexed, modulated into the Quadrature Amplitude Modulation (QAM) domain and sent over Radio Frequency (RF) channels to the nodes where the end users are located [1]. Since several hundreds of TV channels can be present in each incoming GbE signal and only about ten can be carried per RF channel (depending on the QAM modulation used), multiple edge QAM devices are connected through a "daisy chain", for each GbE signal (see Fig. 1).

Standard broadcast TV channels are not only broadcast on the transport network but also on the access network by sending them over broadcast RF channels, through a splitter at the HE, to all nodes simultaneously. Since the installation cost in the access network is mainly determined by the number of out- 
going RF channels on the edge QAM devices, using standard broadcast therefore results in a cheap solution. However, for less popular TV channels, this technology might be very bandwidth consuming on the RF spectrum at the node side.

Switched broadcast TV channels are broadcast on the transport network as well, but only the nodes with at least one user watching a particular channel actually receive that channel, sent over a unicast RF channel. Switched broadcast technologies can therefore be used for less popular TV channels, to save valuable RF spectrum, as channels that are not currently being viewed by at least one subscriber in a node are not streamed to that node. For example, 200 or more broadcast video channels could be offered to the digital cable subscriber while only reserving enough $\mathrm{RF}$ bandwidth for 80 . This is based on the premise that probably no more than 80 different channels will be viewed simultaneously at any one time in a switched broadcast target area, thus achieving statistical gains [4].

Therefore, the goal of this work is to determine the optimal number of TV channels served through standard and switched broadcast technologies, so that the access network installation cost (i.e. the total number of RF channels required at the edge QAM devices) is minimized, while respecting the restrictions on bandwidth spectrum usage.

The remainder of this paper is organized as follows. Section II presents an analytical traffic model, which determines the number of simultaneously watched channels, taking content popularity and user behavior into account. In Section III an access network design tool, based on this traffic model, is described. The influence of different parameters on the network design is studied in Section IV, through a set of simulations. Each time, the results are compared to a standard configuration. Section V concludes this paper and presents ideas for future work.

\section{TRAFFIC MODEL}

This section presents the analytical traffic model that determines the distribution of the number of simultaneously watched TV channels during peak hour, given the number of user requests. This model can later on be used to find the number of unicast (for switched broadcast) and broadcast (for standard broadcast) RF channels required at the edge QAM devices.

\section{A. User Demand}

A typical HFC access network configuration, as shown in Fig. 1, consists of several tens of nodes per HE, each with about $1000 \mathrm{HP}$ (homes passed). To determine the number of user requests per node during peak hour, the total number of users is multiplied by the digital TV market penetration and by the percentage of digital TV users active during peak hour.

The popularity of the available TV channels is represented by a Zipf-like distribution [5]. In a Zipf-like distribution, the popularity of the $i$ th most popular item is proportional to $i^{-\beta}$. We fit this distribution with data from a field trial in Belgium, where $118 \mathrm{TV}$ channels were offered. The corresponding value for the Zipf parameter $\beta$ is about 1.7 (see Fig. 2). 50\% of all requests are made for the most popular channel, $90 \%$ for the top 12 .

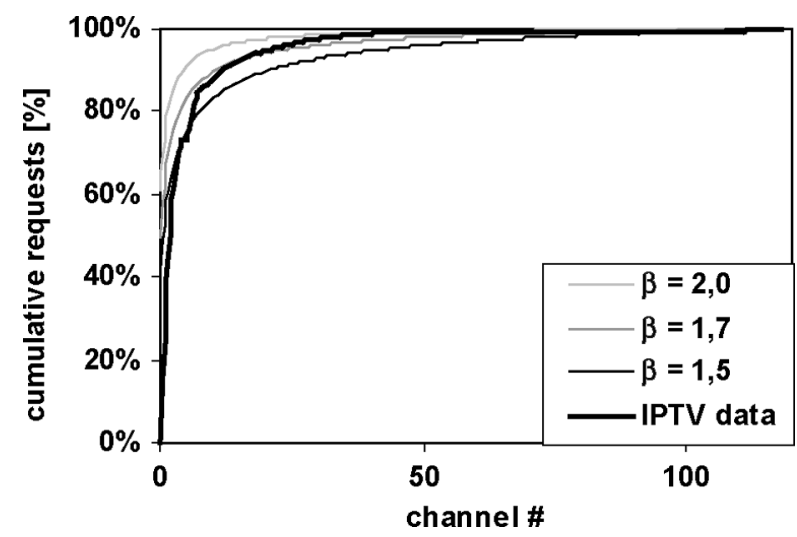

Fig. 2. Cumulative Zipf-like TV channel popularity (ranked), compared for different values of $\beta$.

\section{B. Mathematical Formulation}

The goal of the traffic model is to set up a probability distribution for the number of simultaneously watched TV channels, for a given content popularity and user demand. In other words, we have to find how $R$ simultaneous user requests are distributed over $N$ available TV channels.

1) Variables: We define $N$ variables $X_{n}$ as follows: $X_{n}=0$ if channel $n$ is not requested, $X_{n}=1$ if channel $n$ is requested at least once. When $q(n)$ is the chance that a particular user request is made for channel $n$, the corresponding probabilities for $X_{n}$ are:

$$
\begin{aligned}
& \operatorname{Prob}\left[X_{n}=0\right]=(1-q(n))^{R}=p_{1}(n) \\
& \operatorname{Prob}\left[X_{n}=1\right]=1-(1-q(n))^{R}=1-p_{1}(n)
\end{aligned}
$$

since $\operatorname{Prob}\left[X_{n}=0\right]$ is the chance that all requests are made for one of the $N-1$ other channels than channel $n$ and this chance is $(1-q(n))$ for each of the $R$ individual (and independent) requests. We define $p_{i}$ as the chance that $i$ particular channels are not requested:

$$
\begin{aligned}
p_{1}(n) & =(1-q(n))^{R} \\
p_{2}(j, k) & =(1-q(j)-q(k))^{R}, \quad j \neq k
\end{aligned}
$$

For the above-mentioned Zipf-like channel popularity, we know that $q(n)$ is given by (all channels ranked by popularity):

$$
q(n)=\frac{n^{-\beta}}{\sum_{i=1}^{N} i^{-\beta}}
$$

2) Solution: Our goal is to find the probability distribution for the total number of simultaneously watched TV channels, i.e. channels requested by at least one user:

$$
Y=\sum_{n=1}^{N} X_{n}
$$

$Y$ is the sum of a large number of statistically independent variables ( $N$ variables in total). According to the central limit the- 
orem [6], its distribution can therefore be approximated by a Normal distribution (with mean $\mu$ and variance $\sigma^{2}$ ):

$$
P(y)=\frac{1}{\sigma \sqrt{2 \pi}} e^{\frac{-(y-\mu)^{2}}{2 \sigma^{2}}} .
$$

To calculate $\mu$ and $\sigma^{2}$ we need the following formulas:

$$
\begin{aligned}
E\left[X_{j}\right] & =1 \cdot \operatorname{Prob}\left[X_{j}=1\right]+0 \cdot \operatorname{Prob}\left[X_{j}=0\right] \\
& =\operatorname{Prob}\left[X_{j}=1\right]=1-p_{1}(j) \\
E\left[X_{j}^{2}\right]= & 1 \cdot \operatorname{Prob}\left[X_{j}^{2}=1\right]+0 \cdot \operatorname{Prob}\left[X_{j}^{2}=0\right] \\
= & \operatorname{Prob}\left[X_{j}=1\right]=1-p_{1}(j) \\
E\left[X_{j} X_{k}\right]= & 1 \cdot \operatorname{Prob}\left[X_{j} X_{k}=1\right]+0 \cdot \operatorname{Prob}\left[X_{j} X_{k}=0\right] \\
= & 1-\left(\operatorname{Prob}\left[X_{j}=0\right]+\operatorname{Prob}\left[X_{k}=0\right]\right. \\
& \left.\quad-\operatorname{Prob}\left[X_{j}=X_{k}=0\right]\right) \\
= & 1-\left(p_{1}(j)+p_{1}(k)-p_{2}(j, k)\right)
\end{aligned}
$$

Therefore we find:

$$
\begin{aligned}
\mu_{Y} & =E\left[\sum_{n=1}^{N} X_{n}\right]=\sum_{n=1}^{N} E\left[X_{n}\right]=\sum_{n=1}^{N}\left(1-p_{1}(n)\right) \\
\sigma_{Y}^{2} & =E\left[Y^{2}\right]-E[Y]^{2}=E\left[\left(\sum_{n=1}^{N} X_{n}\right)^{2}\right]-\mu_{Y}^{2} \\
& =E\left[\sum_{n=1}^{N} X_{n}^{2}+2 \sum_{1 \leq j<k \leq N} X_{j} X_{k}\right]-\mu_{Y}^{2} \\
& =\sum_{n=1}^{N} E\left[X_{n}^{2}\right]+2 \sum_{1 \leq j<k \leq N} E\left[X_{j} X_{k}\right]-\mu_{Y}^{2} \\
& =\mu_{Y}-\mu_{Y}^{2}+2 \sum_{1 \leq j<k \leq N}\left(1-p_{1}(j)-p_{1}(k)+p_{2}(j, k)\right)
\end{aligned}
$$

3) Example: Fig. 3 shows that the Normal distribution is indeed a good approximation of the distribution of $Y$. The Normal distribution Fig. 3(b) is very similar to the results of the exact solution, calculated through a brute force computation Fig. 3(a). Since the latter method is computationally heavy, the results are presented for a total of only $20 \mathrm{TV}$ channels $(N=20)$. Fig. 3(c) compares the curves from the exact solution (full line) to the approximated solution (dotted line) and shows a good match (up to $1 \%$ ). When $N$ becomes larger than 20, the match can only become better.

\section{NETWORK DESIGN}

This section describes the methodology of our HFC access network design tool for TV services. The main goal is to find the minimal installation cost (outgoing RF channels at the QAM devices) required to serve all requests, taking a possible restriction on the available RF spectrum at the node side into account. Through an exhaustive method, the optimal choice for each TV channel is made: deliver it using standard or switched broadcast technologies.

\section{A. Input Parameters}

The most important input parameters are listed below. For each parameter a typical (estimated) value is indicated in

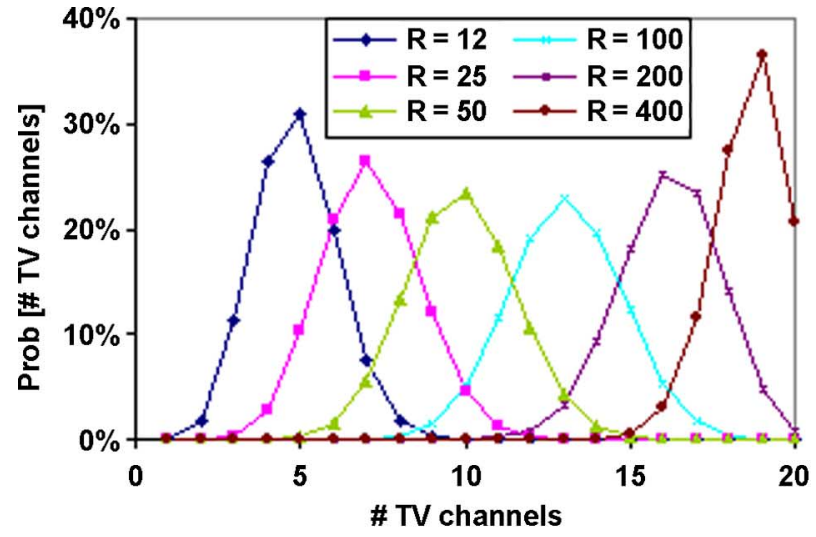

(a)

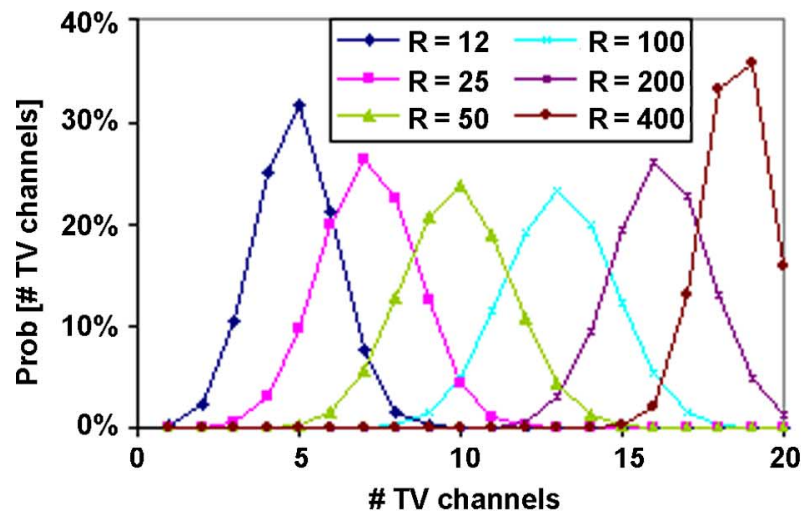

(b)

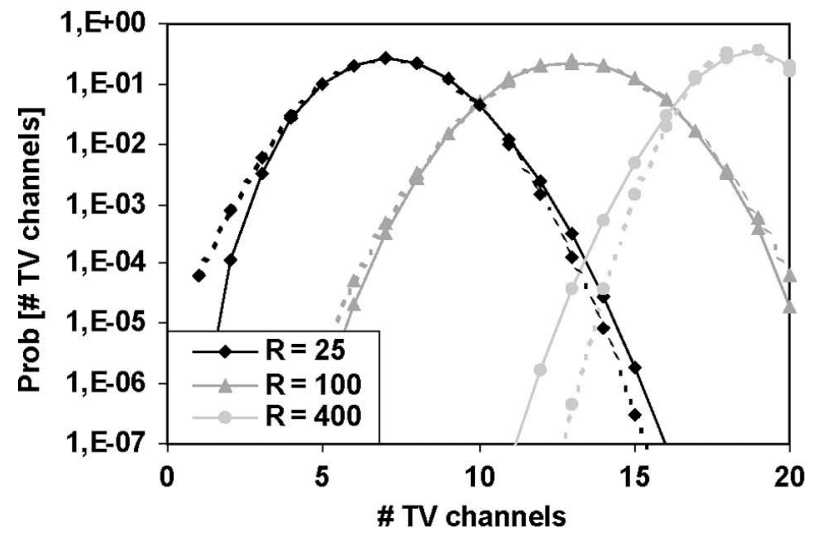

(c)

Fig. 3. Statistical distribution of the number of watched TV channels $(N=$ 20 ), for different values of the total number of user requests $R$ : (a) Exact, (b) Approximated by Normal distribution: (c) Comparison.

brackets, used for the standard configuration to which all other simulations are compared.

For each HE, we need the number of nodes (50), the number of users per node (1000), the digital TV market penetration $(25 \%)$ and the percentage of simultaneous users during peak hour $(40 \%)$, which gives us the maximum number of simultaneous requests per node $R(100)$. Interesting parameters related to the content are the number of TV channels $N$ (200), the Zipf parameter $\beta$ (1.7), the TV channel stream bandwidth (SDTV MPEG-2: $3.8 \mathrm{Mbps})$. We also need the RF channel bandwidth 
(in Europe $8 \mathrm{MHz}, 64 \mathrm{QAM}, 38 \mathrm{Mbps}$ ), the available RF spectrum at each node (10 RF channels) and the cost of one RF output port at the QAM RF devices (1 unit).

Since we approximate the number of simultaneously watched TV programs $Y$ by a Normal distribution (average $\mu$, variance $\sigma^{2}$, we can calculate probability intervals by means of its cumulative distribution function. The following textbook formulas for the Normal distribution are therefore valid [6]:

$$
\begin{aligned}
\operatorname{Prob}[Y \leq \mu] & =0.5 \\
\operatorname{Prob}[Y \leq \mu+1.28 \sigma] & =0.9 \\
\operatorname{Prob}[Y \leq \mu+1.65 \sigma] & =0.95 \\
\operatorname{Prob}[Y \leq \mu+2.33 \sigma] & =0.99 \\
\operatorname{Prob}[Y \leq \mu+3.09 \sigma] & =0.999
\end{aligned}
$$

This percentage of statistical events the system should be capable of serving, can be modified as an input parameter as well ("the size of the uncertainty interval", see Section IV-D). A value of e.g. $99 \%$ (as in the standard configuration) means that of all 100 random events (peak hour situations), on average 99 can be handled by the system, as designed by the tool.

\section{B. Methodology}

The optimal solution is found through an exhaustive method that determines the optimal number of standard broadcast TV channels. First the TV channels are ranked according to popularity, then a variable number of broadcast TV channels is set $(0<n<N)$ and the corresponding design is calculated using the mathematical formulation presented above. As a result, the optimal number of outgoing RF channels (unicast and broadcast) on the QAM devices at the HE is found, as well as the corresponding minimal installation cost.

The value for $n$ that results in the minimal installation cost, while satisfying the restriction on the available RF spectrum at the nodes, is used for the final design. Note that if the $n$ most popular TV channels are sent through standard broadcast, we have to take the following equations into account $(n>0)$ :

$$
\begin{aligned}
p_{1}(j)=0, & \text { if } \mathrm{j} \leq \mathrm{n} \\
p_{2}(j, k)=0, & \text { if } \mathrm{j} \leq \mathrm{n} \text { or } \mathrm{k} \leq \mathrm{n}
\end{aligned}
$$

Fig. 4 shows the influence of the number of broadcast TV channels $(n)$ on the total number of streamed TV channels (through both standard and switched broadcast), for a given number of channels $(N=20)$, user requests $(R=50)$ and content popularity $(\beta=1.7)$.

On average 10 different TV channels (in $99 \%$ of the cases below 13 channels) are requested by the users through switched broadcast $(n=0)$.

Offering more TV channels through standard broadcast obviously decreases the channels sent through switched broadcast, but the total number of TV channels increases. The variability on this total number decreases (to zero for $n=N=20$ ). Note that the curves shown in Fig. 4 are approximations based on the Normal distribution with the same values for the average and variation as the exact solution.

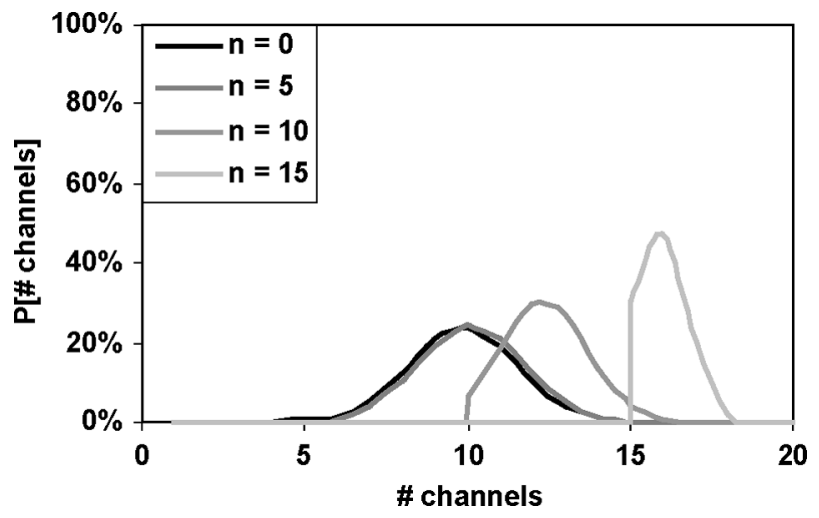

Fig. 4. Distribution of the total number of TV channels streamed to a node, for different numbers n of broadcast TV channels; $N=20, R=50, \beta=1.7$.

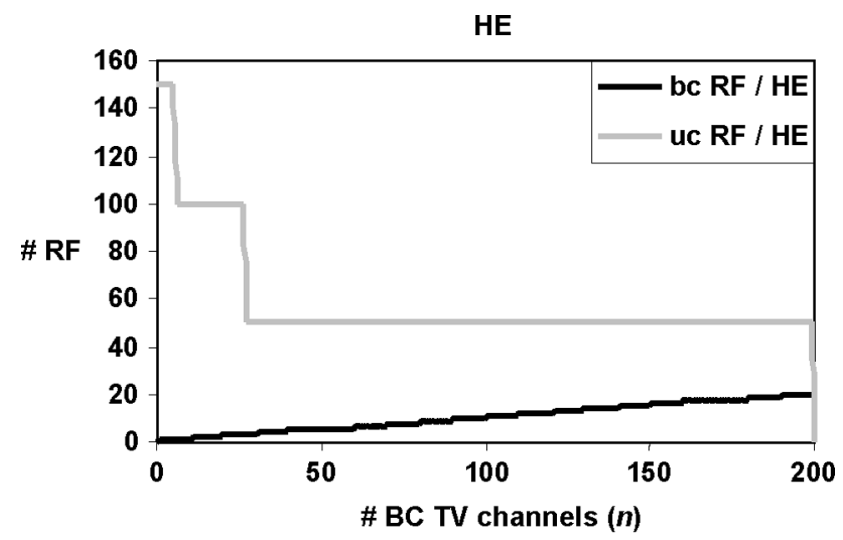

(a)

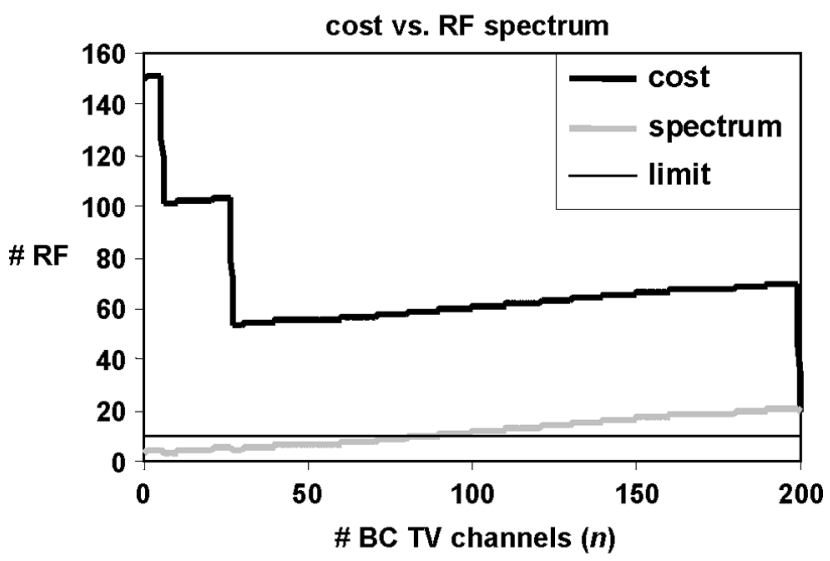

(b)

Fig. 5. Results for the standard configuration, showing (a) the switched broadcast (uc) and standard broadcast (bc) RF channels at the HE and (b) the total installation cost at the HE and the occupied RF spectrum at the node.

\section{Results}

Fig. 5 presents the results for the standard configuration (as in Fig. 1), with the input parameters given above. Fig. 5(a) shows the number of outgoing unicast and broadcast RF channels needed at the $\mathrm{HE}$, for a variable $n$. The number of broadcast (bc) RF channels increases by one every 10 standard broadcast TV channels, the number of unicast (uc) RF channels decreases from 3 to 0 per node (150 to 0 per HE). Fig. 5(b) shows the total installation cost (number of unicast RF + number of broadcast RF at the HE) and the spectrum (number 


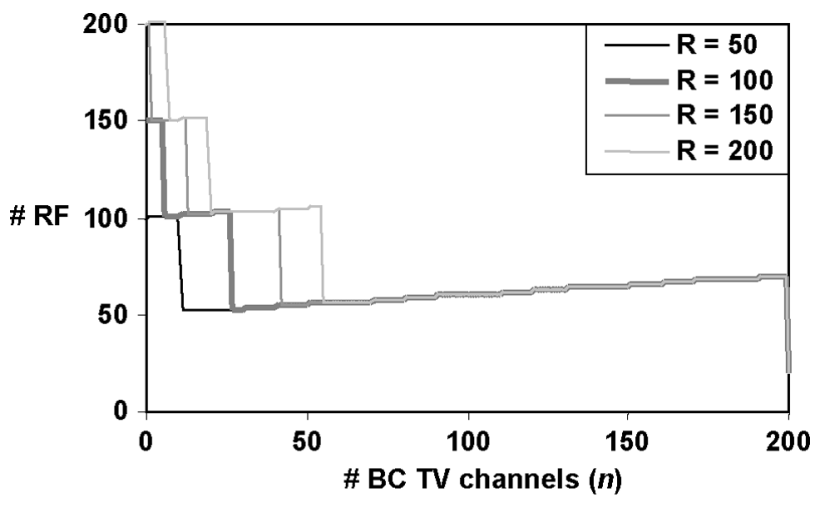

(a)

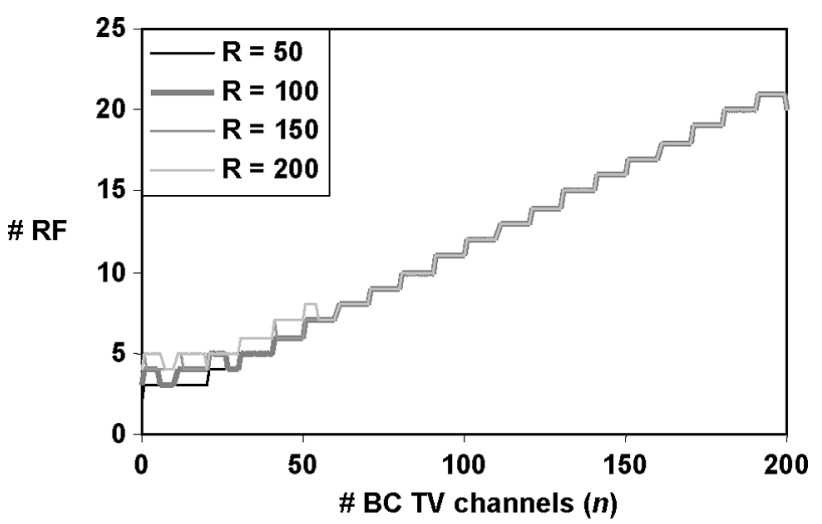

(b)

Fig. 6. Influence of the user demand per node $(R)$ on (a) the total installation cost at the HE and (b) the RF spectrum at the node.

of unicast RF + number of broadcast RF at each node). In this configuration, four local minima for the installation cost can be distinguished, for $n=0($ cost $=50 \cdot 3=150 \mathrm{u}), n=6($ cost $=$ $50 \cdot 2+1=101 u), n=27(\operatorname{cost}=50 \cdot 1+3=53 \mathrm{u})$ and $n=200($ cost $=50 \cdot 0+20=20 u)$.

Since the maximum number of RF channels at each node is limited to 10 , the overall optimum for $n$ is 27 . The corresponding average number of streamed TV channels per node is 32 (27 standard broadcast, 5 switched broadcast), with a $99 \%$ limit of 37 (27 standard broadcast, 10 switched broadcast).

\section{NUMERICAL PARAMETER STUDY}

In this section, we compare the results for the standard configuration in Section III-C (bold curves in the figures below) to those for other configurations, with different values for one parameter at a time. The influence of the restriction on the maximum number of RF channels per node determines which local optimum has to be chosen.

\section{A. Influence of the User Demand}

The influence of changes in the user demand ( $R$ simultaneous requests per node) on the total installation cost is shown in Fig. 6(a). The number of local minima for the installation cost increases from 3 for $R=50$ (at $n=0, n=11$ and $n=200$ ) to 5 for $R=200$ (at $n=0, n=7, n=20, n=55$ and $n=200$ ), but the increase in cost at each minimum is rather small.

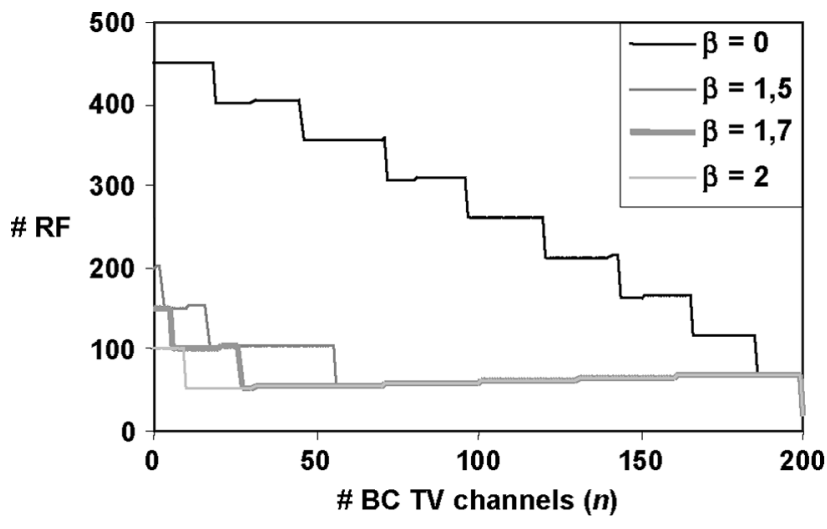

(a)

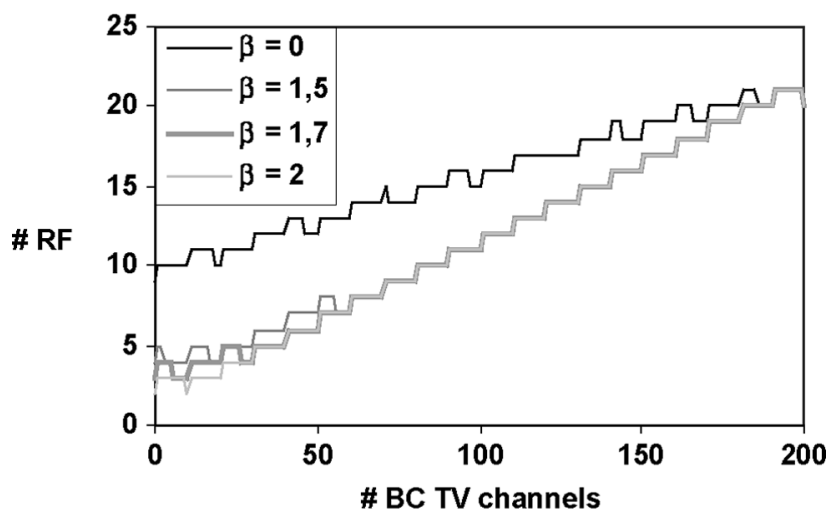

(b)

Fig. 7. Influence of the Zipf parameter $\beta$ for the content popularity on (a) the total installation cost at the HE and (b) the RF spectrum at the node.

This is because of the fact that the main cost is covered by the number of switched broadcast RF channels, which remains the same (e.g. at $50 \cdot 1 u=50 u$ at the optimum). Only the number of standard broadcast RF channels changes, but since these are sent through the splitter (see Fig. 1), the influence on the total installation cost is much smaller.

The influence of the number of standard broadcast RF channels is also visible in the RF spectrum at the nodes Fig. 6(b), but only for lower values.

\section{B. Influence of the Content Popularity}

Changing the popularity distribution of the TV channels also has an impact on the installation cost. The higher the popularity of the top TV channels (high Zipf parameter $\beta$ ), the lower the cost, since all requests for these channels can be served through the "cheaper" standard broadcast service (if sufficient RF channels are available).

Fig. 7 shows the results for small variations around $\beta=1.7$. The most popular channel then receives about $40 \%(\beta=1.5)$, $50 \%(\beta=1.7)$ or $60 \%(\beta=2.0)$ of all requests. $90 \%$ of all requests are made for the top $27(\beta=1.5), 13(\beta=1.7)$ or 6 $(\beta=2.0)$ most popular channels.

When $\beta=0$ (each TV channel is equally popular), Fig. 7(b) shows that the total number of occupied RF channels is always extremely high (at least 9), which increases the total installation cost from 53 to 402 units (minimum at $n=19$ of $50 \cdot 8 \mathrm{u}$ for 


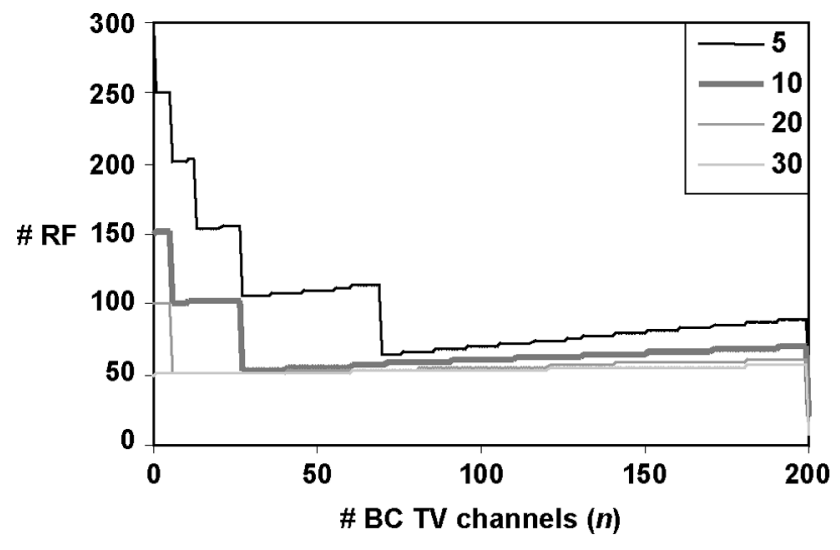

(a)

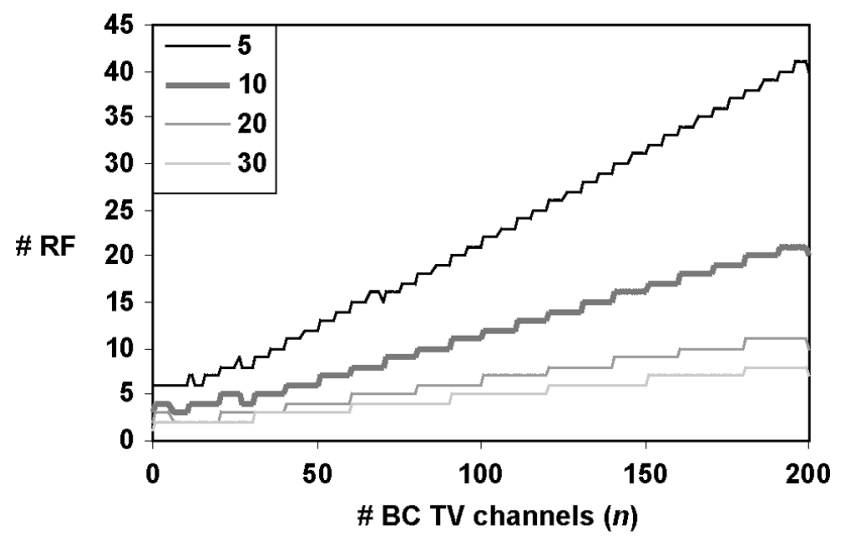

(b)

Fig. 8. Influence of the number of streams per RF channel on (a) the total installation cost at the HE and (b) the RF spectrum at the node.

8 unicast RF channels and $2 u$ for 2 broadcast RF channels), since we need to remain below $10 \mathrm{RF}$ channels per node.

\section{Influence of the Stream Bandwidth}

Changing the quality or format of the streams, e.g. from SDTV MPEG-2 format (3.8 Mbps) to SDTV MPEG-4 (1.6 Mbps) or HDTV MPEG-4 (8.0 Mbps), also has a large impact on the installation cost. Note that the impact of doubling the stream bandwidth is much higher than doubling the user demand, since the latter does not mean that twice as many TV channels will be watched (e.g. contrary to a VoD scenario)! Fig. 8 shows the results for different numbers of streams that can be transported simultaneously in one RF channel. The influence on the RF spectrum is now much more significant. Both the number of local minima and the total installation cost increase quickly. When 30 streams can be transported in one RF channel (e.g. 30 SDTV mpeg 4 streams in one 256 QAM $8 \mathrm{MHz}$ $\mathrm{RF}$ channel), one unicast RF channel per node is enough to serve all users (no standard broadcast TV channels present).

\section{Influence of the Size of the Uncertainty Interval}

The influence of the size of the uncertainty interval $(99 \%$ in the standard configuration) is shown in Fig. 9. The results are similar to those for changes in user demand, but less noticeable.

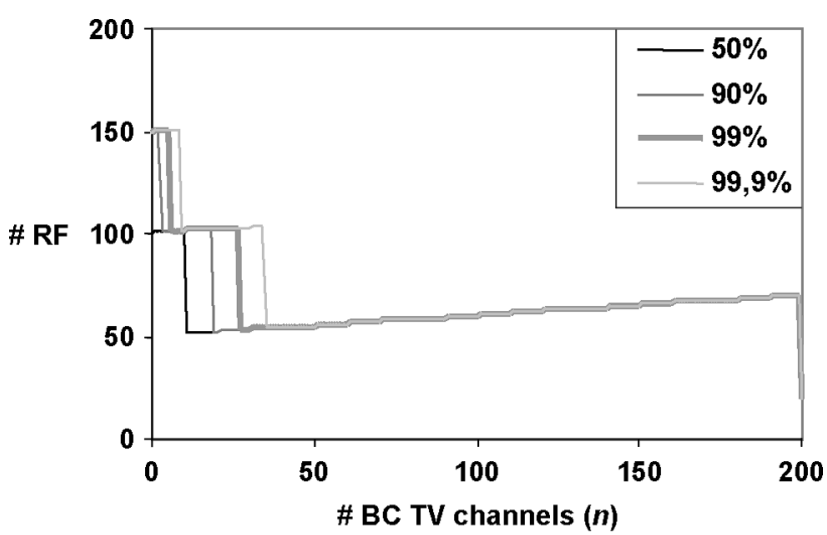

(a)

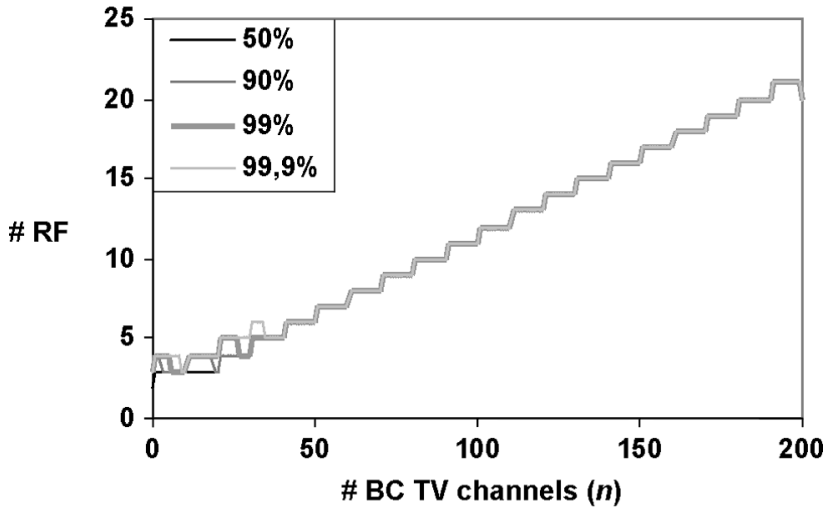

(b)

Fig. 9. Influence of the size of the uncertainty interval on (a) the total installation cost at the HE and (b) the RF spectrum at the node.

\section{E. Conclusion}

The numerical results above show the influence of the most important parameters. The main conclusion is that the cheapest solution would normally be to stream as much TV channels as possible through standard broadcast. The restriction on the available RF spectrum at the node determines whether this solution can be reached or not. If not, one of the local minima has to be chosen. Therefore the number of unicast RF channels must be increased (typically from 1 to 2 or 3 ) and the total installation cost will increase almost proportionally.

\section{CONCLUSIONS}

In this paper, an HFC access network design tool for standard and switched broadcast TV services has been presented. We studied the influence of different traffic and content parameters on different network configurations. The most important parameters have been identified. Currently, traffic models for other interactive services such as VoD, IPTV and Internet access are being developed. These models will be implemented in a tool combining all these services, to further improve the network design for converging HFC access networks through statistical gains and dynamic RF channel allocation. 


\section{ACKNOWLEDGMENT}

This research was performed within the framework of the IBBT-CIcK project. The authors acknowledge the support of the industrial partners in this project. The authors also wish to thank Christophe Lermytte for his valuable input.

\section{REFERENCES}

[1] Broadbus Technologies Inc. White Paper, C. Skarica and J. Selvage, "Network Transport Convergence: Advanced Services Require Advanced Transport Networks," March 2006.
[2] T. Wauters, D. Colle, M. Pickavet, B. Dhoedt, and P. Demeester, "Design of optical content distribution networks for video on demand services," Photonic Network Communications, vol. 11, pp. 253-263, May 2006.

[3] S. V. Vasudevan and P. Brooks, "Planning for and managing the rollout of switched broadcast services," in SCTE 2003 Conference on Emerging Technologies, Miami, Jan. 2003.

[4] N. Sinha and R. Oz, "The statistics of switched broadcast," in Proc. of SCTE 2005 Conference on Emerging Technologies, Huntington Beach, CA, Jan. 2005.

[5] L. Breslau, P. Cao, L. Fan, G. Phillips, and S. Shenker, "Web caching and Zipf-like distributions: Evidence and implications," in Proc. of IEEE Infocom, March 1999, pp. 126-134.

[6] J. K. Patel and C. B. Read, Handbook of the Normal Distribution. New York: Dekker, 1996. 